\title{
Remote activation of microglia and pro-inflammatory cytokines predict the onset and severity of below-level neuropathic pain after spinal cord injury in rats
}

\author{
Megan Ryan Detloff, \\ Center for Brain and Spinal Cord Repair, Neuroscience Graduate Studies Program, The Ohio \\ State University \\ Lesley C. Fisher, \\ Center for Brain and Spinal Cord Repair, Division of Physical Therapy, The Ohio State University \\ Violetta McGaughy, \\ Human Tissue Resource Network, Department of Pathology, The Ohio State University \\ Erin E. Longbrake, \\ Center for Brain and Spinal Cord Repair, Molecular Virology, Immunology and Medical Genetics, \\ The Ohio State University \\ Phillip G. Popovich, \\ Center for Brain and Spinal Cord Repair, Depts. Molecular Virology, Immunology and Medical \\ Genetics and Neuroscience, The Ohio State University

\section{D.Michele Basso} \\ Center for Brain and Spinal Cord Repair, Division of Physical Therapy, Department of \\ Neuroscience, The Ohio State University, 516 Atwell Hall, 453 W. $10^{\text {th }}$ Ave., Columbus, $\mathrm{OH}$ \\ 43210, basso.2@osu.edu, (614) 292-0754
}

\begin{abstract}
Spinal cord injury (SCI) impairs sensory systems causing chronic allodynia. Mechanisms underlying neuropathic pain have been more extensively studied following peripheral nerve injury than after central trauma. Microglial activation, pro-inflammatory cytokine production and activation of p38 MAP kinase pathways may induce at-level allodynia following PNI. We investigated whether midthoracic SCI elicits similar behavioral and cellular responses below the level of injury (lumbar spinal cord; L5). Importantly, we show that anatomical connections between L5 and supraspinal centers remain intact after moderate SCI allowing direct comparison to a well-established model of peripheral nerve injury. We found that SCI elicits below-level allodynia of similar magnitude to at-level pain caused by a peripheral nerve injury. Moreover, the presence of robust microglial activation in L5 cord predicted allodynia in $86 \%$ of rats. Also
\end{abstract}

Correspondence to: D.Michele Basso.

Publisher's Disclaimer: This is a PDF file of an unedited manuscript that has been accepted for publication. As a service to our customers we are providing this early version of the manuscript. The manuscript will undergo copyediting, typesetting, and review of the resulting proof before it is published in its final citable form. Please note that during the production process errors may be discovered which could affect the content, and all legal disclaimers that apply to the journal pertain. 
increased phosphorylation of p38 MAP kinase occurred in the L5 dorsal horn of allodynic rats. For below-level allodynia after SCI, TNF- $\alpha$ and IL- $1 \beta$ increased in the L 5 dorsal horn by 7 dpo and returned to baseline by 35 dpo. Interestingly, IL- 6 remains at normal levels early after SCI and increases at chronic time points. Increased levels of pro-inflammatory cytokines also occurred in the thalamus after SCI-induced allodynia. These data suggest that remote microglial activation is pivotal in the development and maintenance of below-level allodynia after SCI. Fractalkine, a known activator of microglia, and astrocytes were not primary modulators of below-level pain. Although the mechanisms of remote microglial activation are unknown, this response may be a viable target for limiting or preventing neuropathic pain after SCI in humans.

\section{Keywords}

allodynia; 38 ; fractalkine; astrocytes; peripheral nerve injury

\section{INTRODUCTION}

Over two-thirds of patients with spinal cord injury (SCI) have significantly diminished quality of life due to the presence and persistence of neuropathic pain (Widerstrom-Noga et al. 2001; Siddall et al. 2003). Hypersenstivity after SCI is categorized as above-, at- and below-level pain. Clinical and experimental classifications include: allodynia - the perception of an innocuous stimulus as painful; or hyperalgesia - exaggerated, heightened responses to a noxious stimulus (2008). Previously, we and others showed that below-level pain develops after SCI in animal models (Kloos et al. 2005) (Hutchinson et al.

2004;Lindsey et al. 2000; Christensen et al. 1996a;Siddall et al. 1995;Bruce et al. 2002; Yezierski et al. 1993; Hains et al. 2006).

Peripheral nerve injury models are useful for identifying mechanisms of neuropathic pain because aversive responses can be elicited without directly injuring CNS pain pathways. Activated glia (Coull et al. 2005; Tsuda et al. 2003), induction of select intracellular signaling pathways (e.g. p38, (Jin et al. 2003)) and robust increases in cytokine and chemokine synthesis (e.g. TNFa, fractalkine (Milligan et al. 2004;DeLeo et al. 1996;DeLeo et al. 2000; Sweitzer et al. 2001;Raghavendra et al. 2004)) have all been associated with hind paw allodynia after peripheral nerve lesion. Although these cellular and molecular changes are common after SCI, whether they are associated with or are responsible for the onset and maintenance of SCI-induced neuropathic pain is unknown. In fact, few have studied mechanisms of SCI-induced neuropathic pain below the level of injury. Recent reports suggest that robust glial activation occurs at sites caudal to SCI in rats with below-level pain (Hains et al. 2006; Nesic et al. 2005; Peng et al. 2006;Detloff et al. 2004). Additionally, increased expression of TNFa was recently described caudal to the level of SCI and was correlated with short-term allodynia (Peng et al. 2006). To further these observations and provide novel insight to mechanisms of below-level neuropathic pain induced by spinal contusion injury, we tested whether pain behaviors and accompanying glial activation were associated with the upregulation of p38 MAPK and/or fractalkine (CX3CL1). Importantly, these parameters were compared to a conventional model of peripheral nerve injury-induced neuropathic pain where the mechanisms of pain induction and maintenance have been better 
defined. By performing these lesions in parallel, we can begin to understand the cellular and molecular substrates responsible for SCI-induced pain development and maintenance.

Finally, we determined whether pro-inflammatory cytokines are elevated in spinal cord segments removed from the primary site of SCI. Our data show that below-level pain elicited by moderate SCI was associated with a significant increase in microglial activation and proinflammatory cytokines 10 segments below the level of SCI. This work has been reported in abstract form (Detloff et al. 2004;Detloff et al. 2006;Detloff et al. 2007).

\section{METHODS}

\section{Subjects and Surgeries}

Sixty-two adult female Sprague-Dawley rats (205-231g) were randomly assigned to six groups: Naïve ( $\mathrm{n}=12)$, laminectomy control (LAM; $\mathrm{n}=4)$, Mild SCI $(0.5 \mathrm{~mm}$ cord displacement; $\mathrm{n}=5$ ) or Moderate SCI (1.1 mm cord displacement; $\mathrm{n}=29)$, L5 modified spinal nerve ligation $(\mathrm{mSNL})$ with a short ( 14 day; $\mathrm{mSNL} 14 ; \mathrm{n}=4$ ) or long (35 day; mSNL35; $\mathrm{n}=5$ ) survival. The mSNL14 group was included to replicate the study by Tsuda et al. (2003) where activated microglia correlated with allodynic behavior. The mSNL35 rats were included to compare the behavioral and anatomical indicators of allodynia in an established model of neuropathic pain to changes in sensation after SCI. LAM and Mild SCI groups were included as negative controls since neither laminectomy nor mild SCI induce neuropathic pain in rats (Kloos et al. 2005). Rats were housed 2-3 per cage in controlled environment (12 hour light-dark cycle) with food and water at all times. All experimental procedures were approved by The Ohio State University (OSU) Institutional Laboratory Animal Care and Use Committee. Laminectomy and spinal cord contusion surgeries were performed as described previously (Kloos et al. 2005). Briefly, rats were anesthetized with ketamine $(80 \mathrm{mg} / \mathrm{kg}$ )-xylazine $(20 \mathrm{mg} / \mathrm{kg}$ ) and given prophylactic antibiotics (gentocin, $1 \mathrm{mg} /$ $\mathrm{kg}$ ). The T8 lamina was removed, and the spinal cord was rapidly displaced $0.5 \mathrm{~mm}$ or 1.1 $\mathrm{mm}$ for Mild and Moderate groups, respectively, using the OSU Electromagnetic Spinal Cord Injury Device (Jakeman et al. 2000). The incision was closed in layers and $5 \mathrm{cc}$ of sterile saline was administered subcutaneously to prevent dehydration. All rats received antibiotics daily, and bladders were manually expressed 2x/day until a reflex bladder was restored. Vitamin C was given orally daily to prevent urinary tract infections (Behrmann et al. 1992).

The mSNL14 and mSNL35 groups received a modified Kim and Chung (1992) spinal nerve ligation unilaterally at L5 as described by Tsuda et al. (2003;Kim et al. 1992). Briefly, rats were anesthetized with $3.0 \%$ isofluorane in $\mathrm{O}_{2}$. The paraspinal muscles over the L5-S1 spinal processes were removed after which the L6 transverse process was removed. The left L5 spinal nerve was ligated distal to the dorsal root ganglion with 6-0 silk thread and the nerve was cut immediately distal to the ligation to ensure that all axons were disrupted.

At $35 \mathrm{dpo}$, retrograde tract tracing techniques were used for a subset of SCI rats $(\mathrm{n}=3)$ with known pain-like behavior to establish whether anatomical substrates for pain sensation exist after moderate SCI. The distribution and extent of FG-labeled neurons after SCI was compared to normal controls $(\mathrm{n}=3)$. Briefly, a small burr hole was drilled into the skull at $-3.14 \mathrm{~mm}$ from Bregma, $3.3 \mathrm{~mm}$ lateral to midline. A glass micropipette was lowered 5.0

Exp Neurol. Author manuscript; available in PMC 2009 August 01. 
$\mathrm{mm}$ below the surface of the brain, and $200 \mathrm{~nL}$ of $2 \%$ fluorogold (FG) was injected into the ventroposterolateral nucleus of the thalamus. Five days after injection, rats were sacrificed.

\section{Behavioral Measures}

All behavioral testing was conducted by a blinded experimenter preoperatively and at least weekly after SCI or mSNL. Basso, Beattie, Bresnahan Locomotor Rating Scale (BBB) testing was performed preoperatively and at 1,3, 7, 14, 21, 28 and 35 days post operatively (dpo) to confirm lesion severity (Basso et al. 1995).

\section{Mechanical Allodynia}

Rats were habituated to the testing environment for at least 7 days prior to baseline testing and for $20 \mathrm{~min}$ prior to daily threshold testing. Temperature and humidity were monitored to ensure stability across sessions. To determine the degree of tactile sensory changes after SCI or mSNL, von Frey hair monofilaments (VFH, Stoelting Co., Wood Dale, Illinois) were applied to the plantar surface of the hind paw using a modified version of the up-down method (Chaplan et al. 1994). To ensure that all rats could withdraw their hind paw from an unpleasant stimulus, plantar VFH was not initiated after SCI until hind limb weight support recovered according to BBB testing. To maximize distraction and prevent visualization of VFH application to the paw, sugared cereal was offered during testing. Immediate, brisk withdrawal of the paw to VFH application constituted a positive response. The dependence of plantar testing on moderate motor recovery precluded early sensory testing.

In a subset of rats, we tested sensation at $7 \mathrm{dpo}$, prior to the recovery of weight support by applying stimuli to the dorsal L5 dermatome. Rats were acclimated to being loosely wrapped in a hand towel and held with their hind paws lightly resting on a flat surface. The towel occluded vision so that no food distraction was required. Each VFH was applied three times ( $\sim 30 \mathrm{sec}$ apart) in an ascending order to the dorsal surface of the hind paw along the long median axis, just below the middle toe. In dVFH testing, aversive hindpaw withdrawal occurs by rapidly moving the paw posteriorly or externally before flexing the HL. Thus, the stimulus is avoided during the flexor withdrawal phase. Dorsal VFH response thresholds are equivalent to plantar VFH response thresholds (Detloff et al. 2006).

For both plantar and dorsal VFH testing, the response threshold was determined as the lowest force $(\mathrm{g})$ that produced a hind limb withdrawal in at least $50 \%$ of the applications (Hutchinson et al. 2004; Kloos et al. 2005; Lindsey et al. 2000; Chaplan et al. 1994). Hind paw testing order was determined randomly for the naïve, LAM, mild, and moderate SCI groups to minimize order effects. Thresholds for each $\mathrm{HL}$ were averaged for each rat. For the mSNL groups, only the lesioned hind paw was tested.

\section{Histology}

Rats were transcardially perfused with $0.1 \mathrm{M}$ phosphate buffered saline (PBS; $\mathrm{pH}$ 7.4) followed by $4 \%$ paraformaldehyde ( $\mathrm{pH} 7.4$ ) at $35 \mathrm{dpo}$. Spinal cord from L4-L6 was postfixed for one hour in $4 \%$ paraformaldehyde, rinsed overnight in $0.2 \mathrm{M}$ phosphate buffer (PB, $\mathrm{pH}$ 7.4) then cryoprotected in $30 \%$ sucrose before being frozen on dry ice (Popovich et al. 
1997). The L4-L6 block was sectioned at $20 \mu \mathrm{m}$ on a Microm HM505E cryostat and placed on ColorFrost Plus slides (Fisher Scientific).

Analysis of Fluorogold Labeled Neurons-The distribution of FG positive neurons was determined on 3 sections through the L5 segment per rat. Positive labeling consisted of FG-filled cytoplasm and dendrites with bright fluorescence well above background. Labeled cells were mapped on a schematic of the L5 spinal cord as delineated by Molander and colleagues (1984).

Lumbar spinal cord immunohistochemistry-Microglial cells and stress-induced MAP kinases were identified on adjacent sections using the following antibodies: OX42 (microglia, 1:4000; Serotec, Indianapolis, IN), anti-phosphorylated (p)-p38 MAPK antibody (activated p38 MAP kinase; 1:100; Cell Signaling Technology) and anti-glial fibrillary acidic protein (GFAP; astrocytes, 1:8000; Sigma). Dried sections were incubated in 10\% normal serum and $0.1 \%$ Triton-X100 for one hour and incubated for $24-48 \mathrm{~h}$ with primary antibody in a humid chamber at $4^{\circ} \mathrm{C}$. All sections were rinsed in buffer and then incubated in biotinylated secondary antibody (1:400 Vector Laboratories, Burlingame, CA;) for two hours at room temperature. Sections were rinsed in buffer and placed in $6 \%$ hydrogen peroxidemethanol solution to quench endogenous peroxidase activity for 15 minutes. Antibodies were visualized using the Vectastain Elite ABC reagent (Vector Laboratories) and DAB (Vector Laboratories) or SG (Vector Laboratories). Sections were dehydrated, cleared in xylene and coverslipped using Permount (Fisher Scientific). For all immunohistochemical staining, lesioned spinal cord tissue was processed for each antibody eliminating either the primary or secondary antibody steps and replacing with blocking solution to ensure positive labeling on experimental tissue. OX42, p-p38 and GFAP immunoreactivity were quantified using techniques adapted from Popovich et al. (Popovich et al. 1997). Optical density thresholds were manually selected for positively labeled tissue (MetaMorph Premier Imaging System, Universal Imaging Corporation, Downington, PA), and were quantified as the proportional area $(\mathrm{PA})$ of positively stained tissue within a specific region. PA measurements of OX42, p-p38 and GFAP labeling within a lateral and medial region $\left(0.0261 \mathrm{~mm}^{2}\right)$ of the L5 superficial dorsal horns were taken within the focal plane of the image that was maximally contrasted against the background and positively stained cells below the plane of focus. There was no significant difference in section thickness or tissue shrinkage across groups ( $\mathrm{p}>.05)$.

To identify the cells with phosphorylated p38 MAPK, L5 sections were double-labeled with p-p38 and NeuN, Ox42 or GFAP immunofluorescence. Slides were pre-treated with $4 \%$ paraformaldehyde for 30 minutes at room temperature prior to a 1 hour block with $4 \%$ BSA, 0.2\% Triton-X100 in PBS. The NeuN (1:500; Chemicon), Ox42 (1:1000) or GFAP (1:1000) antibody was applied for 2 hours at room temperature and was visualized by goat-antimouse Alexa Fluor 546 (1:500; Molecular Probes, Eugene, OR). To visualize p-p38, antigen retrieval was performed on L5 sections with citric acid ( $\mathrm{pH} 6.0)$ for 1 hour at $90^{\circ} \mathrm{C}$. After which, sections were washed in PBS, and the p-p38 antibody was applied at 1:50 overnight at $4^{\circ} \mathrm{C}$ in a humid chamber. The p-p38 was visualized with goat anti-rabbit Alexa Fluor 488 
(1:1000; Molecular Probes). Evidence of double-labeling was determined using confocal microscopy.

\section{Sample preparation for laser capture microdissection (LCM)}

LCM procedures were conducted as described previously (Kigerl et al. 2007;Longbrake 2007). Briefly, buffers were prepared with DEPC-treated water. Naïve, Moderate spinal cord injured and $\mathrm{mSNL}$ rats at 35 dpo were perfused intracardially with sterile $0.1 \mathrm{M}$ PBS and spinal cords were rapidly dissected and frozen rapidly in supercooled N-methyl butane. Coronal sections were cut on a cryostat (Microm) at $20 \mu \mathrm{m}$ and mounted on RNase-free membrane slides (P.A.L.M.). To delineate cytoarchitecture and facilitate LCM, slides were stained for 30 seconds with hematoxylin (Vector). Spinal dorsal horn laminae I-III were microdissected using the P.A.L.M. Microbeam laser capture system (P.A.L.M. Microlaser Technologies, Germany; (Molander et al. 1984)). Bilateral dorsal horns were collected and pooled from naïve or SCI rats ( $\mathrm{n}=2$ /group). Ligated and non-ligated dorsal horns were identified and separately dissected from mSNL rats.

\section{RNA isolation, amplification and Real-Time PCR}

The RNaqueous Micro kit (Ambion) was used to isolate RNA according to the manufacturer's protocol optimized for LCM samples. Samples were eluted in $15 \mu l$ elution buffer and DNase treated (RNaqueous kit, Ambion). Only good quality RNA samples (verified by gel electrophoresis; Picochip, Agilent Bioanalyzer) were amplified then analyzed. Total RNA ( $5 \mu \mathrm{l}$ samples) was amplified using the SensAmp kit (Genisphere) and cleaned up (MinElute kit; Qiagen, Valencia, CA). cDNA was prepared from this RNA by reverse transcription with SuperScript II and random primers (Invitrogen). The PCR reactions were carried out using $2 \mu \mathrm{l}$ of cDNA, primers specific to the gene of interest (Table 1 ) and SYBR Green master mix (Applied Biosystems) in $20 \mu$ reactions. Samples were run in triplicate; levels of PCR product were measured using SYBR Green fluorescence collected during real time PCR on an Applied Biosystems 7300 system. Melting point analyses were performed for each reaction to confirm single amplified products. Gene expression was extrapolated from standard curves generated concurrently for each gene using a control cDNA dilution series. Expression was normalized to GAPDH. Data are expressed relative to the mean mRNA/GAPDH ratio of laminectomy control for both SCI and mSNL samples.

\section{Protein isolation and quantification}

In a subset of rats with below-level pain ( $\mathrm{n}=20$ ), a time course (Naïve, 7, 21, 35 dpo with 1.1 $\mathrm{mm}$ displacement $\mathrm{SCI}$ ) was completed to determine the phenotypic and functional signature of microglia in the lumbar cord. Rats were perfused with sterile $0.1 \mathrm{M}$ phosphate buffered saline (PBS), and their spinal cords were quickly dissected at L5. Spinal cord blocks were then rapidly frozen in super-cooled isopentane. The segments of the lumbar cord were separated into dorsal and ventral portions before tissue processing. Spinal cord samples were homogenized in RIPA lysis buffer (Pierce) and protease inhibitor cocktail (Roche). After centrifugation at $10,000 \mathrm{~g}$ for 5 minutes, protein concentrations were determined in the cleared lysates by a BSA protein assay (Pierce). Quantitation of cytokines was determined using the SearchLight Proteome Array (Pierce). This array consists of a comprehensive 
multiplexed sandwich ELISA containing 4 capture antibodies spotted on the bottom of a 96well polystyrene microtiter plate. The custom-made arrays were produced by spotting a $2 \times$ 2 pattern using 4 different capture antibodies specific to tumor necrosis factor (TNF)-a, interleukin (IL)-1 $\beta$ IL-6 and CX3CL1 (fractalkine). Each antibody captures a specific protein in the standards and experimental samples once they are added to the plate. The bound proteins were then detected with a biotinylated detection antibody, followed by streptavidin-horseradish peroxidase (HRP) then were visualized with SuperSignal ELISA Femto Chemiluminescent substrate. The luminescent signal produced from the HRPcatalyzed oxidation of the substrate was measured using the SearchLight Imaging System (Pierce). The data is then analyzed using ArrayVision (Pierce). The luminescent signal produced is proportional to the amount of each protein present in the original sample. Concentrations are extrapolated from a standard curve.

\section{Statistical analyses}

Two way repeated measure ANOVAs were performed on all behavioral data (group $\times$ time) as well as proportional area measurements for OX42 and p-p38 upregulation (group $\times$ region) followed by Tukey's post hoc comparisons using the harmonic mean to correct for unequal group sizes. For the mSNL14 and Naïve groups, we used the standard estimation technique of the last value carried forward to perform comparisons between groups at later time points. Real time PCR data were analyzed via one-way ANOVA with Bonferroni's post-hoc analysis and significance is reported relative to laminectomy control samples. ELISA data were analyzed with one-way ANOVAs. Regression analysis was completed to determine the relationship between allodynia and the percent of microglial activation normalized to uninjured Naïve and LAM levels. A $\chi^{2}$ analysis was used to evaluate incidence of allodynia in each group. To determine the significant difference in sensory thresholds to the percent microglial activation, we generated a logarithmic regression using endpoint VFH scores of the left hind limbs and the normalized values of microglial activation in the superficial dorsal horn. We also calculated the amount of microglial activation that accurately predicted allodynia of the hind paw based on sensitivity and specificity statistical tests. Sensitivity was computed as the proportion of all rats with allodynia of the hindpaw that had $\geq 2$ fold increase in microglial activation, while specificity was determined as the proportion of all rats with no allodynia that had $<2$ fold increase in microglial activation. All correlations were made with Spearman Rank Test. Means and standard error of the mean (SEM) are reported throughout.

\section{RESULTS}

\section{Moderate $\mathrm{SCl}$ and $\mathrm{mSNL}$ elicit similar allodynic behavior}

Mechanical Allodynia-Although the mode and site of nerve trauma are markedly different between SCI and mSNL groups, by 21 dpo, all rats in the mSNL35 and the Moderate SCI groups exhibited allodynia of similar magnitude (Fig. 1). No rats demonstrated overt evidence of spasticity prior to or during sensory testing such as clonus, sustained anti-gravity posturing of the $\mathrm{HL}$ and resistance to velocity-dependent, passive joint movement. Importantly, allodynia persisted for the duration of the study in these groups compared to all other groups $\left(\chi^{2}=30.08 \mathrm{df}=4, \mathrm{p}<0.001\right)$. The magnitude and duration of 
SCI-induced allodynia was comparable to previous studies (Yezierski et al. 1993;

Christensen et al. 1996;Hulsebosch et al. 2000;Bruce et al. 2002;Lindsey et al.

2000; Hutchinson et al. 2004;Kloos et al. 2005;Hains et al. 2006). The development of SCIinduced allodynia was dependent on injury severity as reported by Kloos and colleagues (2005). Naïve, LAM, Mild SCI and the contralateral paw of mSNL groups had normal sensation throughout the study. The onset of allodynia after moderate SCI could not be detected with plantar testing techniques until 14-21 dpo but was apparent at 7 dpo with dorsal testing (Fig. 6A). A positive response to plantar testing requires greater motor recovery and delays the detection of allodynia.

\section{Axons span the lesion epicenter connecting the VPL of the thalamus and the L5 spinal cord}

After SCI, damage to the dorsal funiculus and anterolateral tract may disrupt sensory afferent transmission to the brain. In the absence of supraspinal transmission, the allodynic responses that we observed could be interpreted as a segmentally controlled, hyper-reflexic phenomenon. To determine whether ascending sensory pathways are spared after moderate SCI, we injected $2 \%$ fluorogold into the VPL of the thalamus bilaterally. Moderate neuronal labeling occurred in the deep dorsal horns at L5 of rats with SCI-induced allodynia (Fig. 2F) compared to naïve controls (Fig. 2E). These thalamus-projecting neurons occupied regions of wide dynamic range neurons (Laminae IV and V). The distribution of spared spinothalamic neurons occurred in normal regions with no obvious loss of specific populations in the superficial or deep dorsal horn (Fig. 2E, F). While some spinothalamic axons are lost after moderate SCI, a similar extent of sparing occurs across rats (Fig. 2F).

\section{Cellular and molecular mechanisms of pain behavior after mSNL and Moderate SCI}

Microglial activation-To define potential mechanisms of the pain behavior noted above, we evaluated cellular and molecular changes in the L5 dorsal horns. Given an equivalent magnitude of allodynia for SCI and mSNL, we used the mSNL model as a positive control to identify cellular and molecular mechanisms of SCI-induced allodynia. Robust microglial activation was evident qualitatively and quantitatively after Moderate SCI and ipsilateral mSNL (Fig. 3D-G) but not in Naïve, LAM or Mild SCI groups (Fig. 3B, C). After Mild SCI, a few phagocytic microglia and/or monocytes were noted along the ventromedian fissure and within the dorsal columns, but not the gray matter of the L5 spinal cord. Microglial activation remained at control levels contralateral to mSNL (Fig. 3G right). Consistent with the increased microglial activation observed via immunohistochemistry, we found expression of CD11b mRNA to be markedly increased in the spinal cord dorsal horn after moderate SCI and mSNL35 (Fig. 3I).

The degree of microglial activation in the superficial dorsal horn was positively associated with the severity of allodynia after Moderate SCI or mSNL (Fig. $3 \mathrm{H} ; \mathrm{r}^{2}=0.829, \mathrm{p}<0.01$ ). As microglial activation increased, greater pain-like behavior developed (Fig. $3 \mathrm{H}$ ). Rats were distributed into two general groups - those with resting microglia and those with a $\geq 2$ fold increase microglial activation compared to naïve rats. Using statistical measures of sensitivity and specificity, we determined how well factors such as activated microglia identify neuropathic pain or normal sensation. High sensitivity showed that high levels of

Exp Neurol. Author manuscript; available in PMC 2009 August 01. 
activated microglia predicted pain-like behavior with $86 \%$ accuracy. High specificity indicated that little or no microglial activation predicted normal sensation in $92 \%$ of rats.

p-38 MAP kinase-Phosphorylated p38 MAP kinase (p-p38 MAPK) localized to the nucleus of cells within the L5 superficial dorsal horn and increased after moderate SCI (Fig. $4 \mathrm{~A}-\mathrm{C})$. The majority of pp38 positive cells in the dorsal horn were neurons not microglia (Fig. 4E-G). Microglia and astrocytes were rarely or never p-p38 MAPK positive, respectively (data not shown). Similar co-labeling in neurons but not glia occurred in the mSNL groups. Quantitative analysis showed significantly greater p-p38 MAPK in the Moderate SCI, mSNL35 and mSNL14 groups compared to Naïve, LAM and Mild SCI controls (Fig. 4C, p<.05). Increased p-p38 MAPK correlated with the development of allodynia; as p-p38 increased, greater pain-like behavior developed $\left(r^{2}=0.63, p<0.01\right.$; Fig. 4D). Rats were distributed into two groups-those with normal p-p38 levels and those in which p-p38 was increased $>2$ fold above Naïve (Fig. 4D). Using sensitivity measures, we predicted pain-like behavior based on the degree of p38 MAP kinase phosphorylation with $87 \%$ accuracy. Normal sensory thresholds were predicted with $93 \%$ accuracy when p-p38 levels were normal.

Astrocyte activation-Neither moderate SCI nor SNL caused a significant change in astroglial morphology (GFAP) in the dorsal horns (Fig. 5A, p>.05). Indeed, astrocytes exhibited a resting phenotype comparable to naïve controls within the grey matter of moderate SCI at 35dpo, mSNL14 and mSNL35 groups. Curve estimation and regression analysis determined no significant correlation between the development of allodynia and the astrocyte response within the L5 superficial dorsal horn $\left(r^{2}=0.02 ; p>.05\right)$. In contrast to unchanged protein levels of GFAP, we found increased levels of GFAP mRNA in the L5 dorsal horn at 35 dpo compared to naïve levels for both moderate SCI and mSNL35 groups (5B).

\section{Pro-inflammatory cytokine production in remote regions from $\mathrm{SCl}$ is associated with below-level neuropathic pain}

In peripheral models of neuropathic pain, microglial activation is associated with an increase in proinflammatory cytokines and chemokines in the L5 dorsal horn (Tanga et al. 2004;Raghavendra et al. 2004;DeLeo et al. 2004; Milligan et al. 2004;Johnston et al. 2004). To determine if a similar relationship exists after SCI, we measured changes in proinflammatory cytokines and fractalkine in the L5 dorsal quadrant of the spinal cord of Moderate SCI rats over the time course of noted changes in pain behavior (7,21 and 35 days post-injury; see Fig. 6). In the L5 spinal cord, TNF- $a$ and IL-1 $\beta$ were elevated at 7 dpo and $21 \mathrm{dpo}$, and returned to baseline at $35 \mathrm{dpo}$ (Fig. 6B, D). Conversely, IL-6 did not increase until 21 and 35 dpo (Fig. 6F). TNFa, IL-1 $\beta$, and IL-6 each show a significant relationship to pain-like behavior (Fig. 6C, E, G, p<.05) suggesting that these cytokines may work symbiotically in the lumbar spinal cord to initiate and then maintain pain-like behavior of the hind paw after SCI. Surprisingly, fractalkine protein which is elevated in peripheral models of pain (Milligan et al. 2004), was unchanged at 7, 21 or 35 days after SCI in the L5 dorsal horn (Fig. 7A). Fractalkine gene expression (CX3CL1; Fig. 7B) at 35 dpo was significantly reduced in the superficial dorsal horn compared to naïve controls with LCM/ 
rtPCR. Fractalkine receptor mRNA (CX3CR1), a molecule predominantly localized to CNS microglia (Harrison et al. 1998), was increased in the dorsal horn after moderate SCI or mSNL (Fig. 7C).

\section{DISCUSSION}

The current work characterizes the cellular and molecular responses in the L5 dorsal horn of rats with below-level pain after SCI. We show that SCI of sufficient severity induces allodynic-like behavior and robust microglial activation 10 segments below the injury site. Importantly, we are the first to describe the contusion-induced inflammatory microenvironment at L5 where these microglia reside. Elevation of the proinflammatory cytokines TNF- $a$, IL-1 $\beta$, and IL-6 occurred in the lumbar spinal cord where below-level allodynia is processed. We speculate that the cytokine microenvironment remote to SCI may modulate below-level allodynia through sensitization of second order neurons in the dorsal horn. That elevated p38 localized to these neurons lends support to this theory. Moreover, we directly compared the SCI-induced cellular and molecular responses to $\mathrm{mSNL}$, a model in which mechanisms of pain induction and maintenance have been better defined.

\section{Evidence for Below-Level Pain not Hyperreflexia}

Allodynia is a painful perception to innocuous stimulation and incorporates endocrine, autonomic, emotional, aversive, and arousal responses. Although evidence of allodynia after $\mathrm{SCI}$ is interpreted from aversive (motor-dependent) reactions to small caliber monofilaments, these responses might represent increased hyperreflexia due to loss of descending supraspinal control of sensory afferents. Based on our present data, we propose that hind limb withdrawal to fine tactile stimulation leads to supraspinal perception of the stimulus and thus, represents below-level allodynia. Indeed, we show that neuroanatomical substrates reach supraspinal pain nuclei (VPL) from below-level spinal segments which display allodynic-like responses after SCI (Fig. 2; (Gerke et al. 2003; Hofstetter et al. 2003)). Thus, substrates are in place after moderate SCI to convey VFH sensory information to supraspinal nuclei responsible for pain perception. Sensitization and spontaneous firing of thalamic neurons are induced by a small number of spared spinothalamic axons after SCI lending further support that aversive behaviors reflect supraspinal pain perception (Zhao et al. 2007b;Hubscher 2006) (Gerke et al. 2003). Moderate SCI produced an equivalent severity of allodynic-like behavior to a known model of supraspinal pain, mSNL, suggesting that similar CNS systems are responsible for pain perception after SCI (Fig. 1; (Dowdall et al. 2005; Kim et al. 1997)).

\section{Inflammatory Mechanisms of Below-Level Pain after SCI}

Robust microglial activation at the site of SCI is well-established (Popovich et al. 1997; Sroga et al. 2003). However, the onset and severity of activated microglia rostral or caudal to the SCI and their impact on functional behavior have received little attention (Koshinaga et al. 1995; Hains et al. 2006). We show that by 7 dpo, robust activation of microglia was evident 10 segments caudal to the site of moderate SCI and was sustained for at least 35 days. Profound activation of microglia and p-38 occurred concomitant with increased pro-inflammatory cytokine production. We have modest evidence of inflammation 
within the brain (VPL) in SCI rats with allodynia which agrees with others (Zhao et al. 2007b). Importantly, these remote indices of inflammation and gliosis correlated with the severity of below-level allodynia. Thus, SCI-induced allodynia likely has widespread cellular mechanisms that are at least in part based on neuroinflammatory cascades.

Microglial and p38 activation were specific features of below-level allodynia and were not a general response to SCI. We did not find elevated levels of activated microglia or p-p38 at L5 after mild SCI or on the non-ligated side of the cord after mSNL. Thus, the severity of SCI must reach a threshold before remote cellular and molecular changes are initiated and allodynia occurs (Hao et al. 1991; Yezierski et al. 1993; Yezierski 2000). Based on factors at the epicenter, we previously estimated the threshold to be $\sim 80 \%$ tissue loss which is in agreement with axonal line counts (Kloos et al. 2005;Hubscher 2006). Whether lesion severity and/or factors emanating from the lesion epicenter produce remote gliosis is unknown.

Current findings establish that cellular and molecular responses within the allodynic dermatome well-below the SCI are an accurate, predictive threshold of allodynia. Indeed, a two-fold increase in microglial or p38 activation identified allodynia with $86 \%$ accuracy. When cellular responses fell below this threshold, we ruled out allodynia with $96 \%$ accuracy. Others have shown that activated microglia are sufficient to induce allodynia in normal rats (Coull et al. 2005; Tsuda et al. 2003). Our work identifies the minimum cellular thresholds associated with below-level allodynia after SCI. Thus, remote activation of microglia, p38 and pro-inflammatory cytokines are likely sensitive biomarkers of neuropathic pain. Whether they can be used to diagnose clinical or experimental allodynia using minimally-invasive techniques requires further study. Regardless, they may be effective therapeutic targets. Pharmacologic reduction of microglial activation attenuated peripheral nerve injury (Ledeboer et al. 2005) and SCI-induced pain (Hains et al. 2006; Zhao et al. 2007b;Zhao et al. 2007a). That below-level pain is induced by activated microglia after SCI raises an important question as to the factors which produce behavioral changes.

\section{A complex network of neurons \& microglia regulate below-level allodynia}

After peripheral nerve injury, activated microglia modulate sensation via production of proinflammatory cytokines (Raghavendra et al. 2003; Raghavendra et al. 2004; Tanga et al. 2004; Tanga et al. 2005). Our study extends these findings to SCI-induced allodynia. We show time-dependent expression of TNFa, IL- $1 \beta$ and IL- 6 in the lumbar cord after midthoracic SCI correlated to the severity of below-level allodynia. We speculate that early increases in TNF- $\alpha$ and IL-1 $\beta$ may promote the induction of below-level allodynia. Conversely, late increases in IL-6 may assist in pain maintenance. That TNF- $\alpha$ and IL-1 $\beta$ initiate transcription of IL-6 (Zhang et al. 1990) lends further support to the maintenance role of IL-6.

Given that cytokine expression results from a complex interaction between neurons, astrocytes and microglia, it seems likely that this network collectively contributes to pain onset and maintenance. A variety of lesion specific signals at the epicenter could induce cytokine expression in the lumbar cord. Glutamate excitotoxicity after SCI may promote production of the chemokine CCL21, a potent microglial activator, which is vesicilized and 
released in remote regions (de Jong et al. 2005;McAdoo et al. 1999;Liu et al. 1991). Indeed, Zhao et al. (Zhao et al. 2007b) showed that regulation of CCL21-mediated activation of microglia in the VPL controlled the development of below-level pain after SCI. Whether CCL21 is responsible for lumbar microglial activation remains unknown. Another potent inflammatory mediator may be toll-like receptor 4 , since it is activated after SCI and regulates pro-inflammatory cytokines as well as allodynia (Kigerl et al. 2007; Tanga et al. 2005). These signals induce proinflammatory cytokine production by activated microglia in the lumbar cord (Fig. 6). We postulate that these cytokines act as potent neuromodulators which sensitize nociceptors, primary afferents and wide dynamic range neurons in the lumbar dorsal horn. Such neuromodulation has been identified for TNFa and IL-6 (Davies et al. 2006;Brenn et al. 2007). It remains to be seen if temporal expression of specific cytokines differentially alter the firing threshold of dorsal horn neurons in the allodynic dermatomes. Cytokine-mediated neuronal excitability appears to be accompanied by elevated p38 MAP kinase (Fig. 4) and NF- $\mathrm{kB}$ which in turn produce greater cytokine transcription (Zhao et al. 2001; Viviani et al. 2004). Several reports suggest that the p38 MAP kinase pathway is activated in SCI-induced pain (Crown et al. 2005;Crown et al. 2006;Peng et al. 2006; Hains et al. 2006). Recently, activated p38 in neurons was shown to be a more potent regulator of allodynia than p38 expression in microglia after SCI (Gwak et al. 2007). Further studies will help delineate whether activated p38 alone is sufficient to induce below-level allodynia.

\section{Other Potential Factors in SCl-Induced Below-Level Allodynia}

To elucidate whether additional cellular mechanisms induced below-level allodynia after SCI, we examined factors associated with exacerbation of allodynia after peripheral nerve injury or SCI - fractalkine (CX3CL1) and astrocytic activation (Tawfik et al. 2007; Tanga et al. 2004;Lindia et al. 2005;Takeda et al. 2004;Macias et al. 2006;Nesic et al. 2005;Milligan et al. 2004). We reasoned that cellular mechanisms of below-level allodynia would be most apparent within cord regions associated with the affected dermatomes. By examining cellular responses 10 segments caudal to the injury, we have greater assurance that any changes would be related to pain rather than lesion neuropathology. Reactive astrocytes are prominent at the injury epicenter (Nesic et al. 2005; Reier et al. 1988). However, we found no evidence of astrocyte hypertrophy (GFAP labeling) in the lumbar cord after moderate SCI or mSNL. Nesic et al. (2005) showed that SCI generates elevated GFAP protein in the lumbar cord up to 4 weeks post-injury which parallels increases in GFAP gene expression. However, at later time points post SCI, GFAP protein returned to basal levels within the lumbar gray matter, in agreement with our findings ((Nesic et al. 2005); Fig. 5A). We show an intriguing disconnect between GFAP gene expression (Fig. 5B) and its translation to protein at these chronic time points after SCI (Fig. 5A). The failure of gene transcription to translate into protein in astrocytes is not new. In fact, increases in GFAP mRNA and protein coincide early after peripheral nerve injury but protein levels return to baseline at late time points ( 28 or 42 days) or with increased age (Kane et al. 1997;Gilmore et al. 1998; Tanga et al. 2006). Molecular changes in GFAP may not be an effective marker of neuropathic pain at time points beyond 28 days. 
The role of reactive astrocytes in neuropathic pain remains uncertain given the dissociation between pain behavior and astrocyte activation (Zhuang et al. 2006). Several studies including ours demonstrate profound neuropathic pain despite the absence of reactive astrocytes after peripheral nerve injury or SCI ((Tanga et al. 2006; Stuesse et al. 2001;Tawfik et al. 2007); Fig. 5). Furthermore, recovery from allodynia occurs without noticeable changes in astrocyte activation (Zhuang et al. 2006). Taken together, astrocytes appear to be a marker of central or peripheral nerve injury rather than a sensitive indicator of allodynia. However, a time course analysis is required to identify the early role of astrocyte activation in mediating the development of neuropathic pain after peripheral nerve injury or SCI.

Fractalkine is a neuronally-derived chemokine implicated in the potentiation of pain states (Milligan et al. 2004). LCM analysis of mRNA for fractalkine and its receptor CX3CR1 (found predominantly on microglia (Harrison et al. 1998), revealed model-dependent differences in gene expression. Specifically, we noted a decrease in fractalkine mRNA after SCI but not after mSNL. Reduced fractalkine mRNA caudal to the lesion may indicate damage or death of fractalkine expressing neurons. Interestingly, we noted a robust increase in CX3CR1 in both models albeit to a greater extent after mSNL. Changes in CX3CR1 mRNA paralleled those of CD11b mRNA suggesting that activated microglia upregulate expression of receptors for fractalkine. Despite the upregulation of CX3CR1, fractalkine is probably not responsible for SCI-induced microglial activation as levels of fractalkine protein was unchanged in rats with SCI-induced pain.

Conclusion-This study identifies remote microglial and p38 activation as predictors of below-level allodynia after SCI. It also specifies the onset and time course of proinflammatory cytokine cascades that may be responsible for perpetuating this allodynia. As pain is a multifaceted perception, it is unlikely that microglial modulation is the sole mediator of allodynia. Indeed, several other mechanisms of below-level pain have been elucidated by this lab and others(Christensen et al. 1996b;Christensen et al. 1997; Hains et al. 2002; Hains et al. 2006;Laughlin et al. 2000; Mills et al. 2002;Nesic et al. 2005).

Ameliorative treatment of neuropathic pain will likely require combinatorial strategies to address multiple factors. Therefore, it is as important to identify mechanisms of allodynia as it is to rule out non-contributing factors.

\section{ACKNOWLEDGMENTS}

Our sincerest thanks to Patricia Walters, A. Todd Lash, Wenmin Lai, Ming Wang, Anne D. Kloos, Zachary Kloos, Richa B. Tripathi, and Emily Hoschouer of The Ohio State University's Center for Brain and Spinal Cord Repair (CBSCR) for their assistance with animal care, surgery and behavioral procedures. Support for this work was contributed by NINDS \#NS43798 (DMB), NINDS \#NS37846 (PGP), F31 \# NS058138 (MRD) and P30NS045758.

\section{Reference List}

1. International Association for the Study of Pain. Website 11-21-2005. 2008

2. Basso DM, Beattie MS, Bresnahan JC. A sensitive and reliable locomotor rating scale for open field testing in rats. J.Neurotrauma. 1995; 12:1-21. [PubMed: 7783230]

3. Behrmann DL, Bresnahan JC, Beattie MS, Shah BR. Spinal cord injury produced by consistent mechanical displacement of the cord in rats: Behavioral and histologic analysis. J.Neurotrauma. 1992; 9:197-217. [PubMed: 1474608] 
4. Brenn D, Richter F, Schaible H-G. Sensitization of unmyelinated sensory fibers of the joint nerve to mechanical stimuli by interleukin-6 in the rat: An inflammatory mechanism of joint pain. Arthritis Rheum. 2007; 56:351-359. [PubMed: 17195239]

5. Bruce JC, Oatway MA, Weaver LC. Chronic pain after clip-compression injury of the rat spinal cord. Exp.Neurol. 2002; 178:33-48. [PubMed: 12460606]

6. Chaplan SR, Bach FW, Pogrel JW, Chung JM, Yaksh TL. Quantitative assessment of tactile allodynia in the rat paw. J.Neurosci.Methods. 1994; 53:55-63. [PubMed: 7990513]

7. Christensen MD, Everhart AW, Pickelman JT, Hulsebosch CE. Mechanical and thermal allodynia in chronic central pain following spinal cord injury. Pain. 1996a; 68:97-107. [PubMed: 9252004]

8. Christensen MD, Hulsebosch CE. Spinal cord injury and anti-NGF treatment results in changes in CGRP density and distribution in the dorsal horn in the rat. Exp.Neurol. 1997; 147:463-475. [PubMed: 9344570]

9. Christensen MD, Supowit SC, DiPette DJ, Hulsebosch CE. Enahnced gene espression of CGRP and GAP-43 in dorsal root ganglia by spinal hemisection and nerve growth factor. J.Neurotrauma. 1996b; 13:602.

10. Coull JAM, Beggs S, Boudreau D, Boivin D, Tsuda M, Inoue K, Gravel C, Salter MW, De Koninck Y. BDNF from microglia causes the shift in neuronal anion gradient underlying neuropathic pain. Nature. 2005; 438:1017-1021. [PubMed: 16355225]

11. Davies AL, Hayes KC, Shi R. Recombinant human TNFalpha induces concentration-dependent and reversible alterations in the electrophysiological properties of axons in mammalian spinal cord. J.Neurotrauma. 2006; 23:1261-1273. [PubMed: 16928184]

12. de Jong EK, Dijkstra IM, Hensens M, Brouwer N, van Amerongen M, Biem RSB, Boddeke HWGM, Biber K. Vesicle-mediated transport and release of CCL21 in endangered neurons: a possible explanation for microglia activation remote from a primary lesion. J.Neurosci. 2005; 25:7548-7557. [PubMed: 16107642]

13. DeLeo JA, Colburn RW, Nichols M, Malhotra A. Interleukin-6-mediated hyperalgesia/allodynia and increased spinal IL-6 expression in a rat mononeuropathy model. J.Interferon Cytokine Res. 1996; 16:695-700. [PubMed: 8887053]

14. DeLeo JA, Rutkowski MD, Stalder AK, Campbell IL. Transgenic expression of TNF by astrocytes increases mechanical allodynia in a mouse neuropathy model. NeuroReport. 2000; 11:599-602. [PubMed: 10718321]

15. DeLeo J, Tanga FY, Tawfik VL. Neuroimmune Activation and Neuroinflammation in Chronic Pain and Opioid Tolerance/Hyperalgesia. The Neuroscientist : a review journal bringing neurobiology, neurology and psychiatry. 2004; 10:40-52.

16. Detloff, MR, Fisher, LC, Kloos, ZA, Kloos, AD, Miller, EL, McGaughy, V, Popovich, PG, Basso, DM. Abstract Viewer/ Itinerary Planner. Washington, DC: Society for Neuroscience; 2004. Neuropathic pain after spinal cord injury and its relationship to glia and ATP receptor upregulation.

17. Detloff MR, Fisher LC, McGaughy V, Longbrake E, Popovich PG, Basso DM. Remote activation of microglia and pro-inflammatory cytokines indicate below-level neuropathic pain after spinal cord injury. Exp.Neurol. 2007

18. Detloff, MR, Fisher, LC, Song, X, Basso, DM. Abstract Viewer/Itinerary Planner. Atlanta, GA: Society for Neuroscience; 2006. SCI-induced neuropathic pain: mechanisms of microglial activation in segmental and supraspinal centers.

19. Dowdall T, Robinson I, Meert TF. Comparison of five different rat models of peripheral nerve injury. Pharmac.Biochem.Behav. 2005; 80:93-108.

20. Gerke MB, Duggan AW, Xu L, Siddall PJ. Thalamic neuronal activity in rats with mechanical allodynia following contusive spinal cord injury. Neuroscience. 2003; 117:715-722. [PubMed: 12617975]

21. Gilmore SA, Kane CJ. Microglia, but not astrocytes, react to sciatic nerve injury in aging rats. Brain Res. 1998; 806:113-116. [PubMed: 9739119]

22. Gwak, Y, Unabia, C, Hulsebosch, CE. Neuroscience Meeting Planner. San Diego, CA: Society for Neurosicence; 2007. Activation of neuronal, not glial, p-38 MAPK maintains hyperexcitability of

Exp Neurol. Author manuscript; available in PMC 2009 August 01. 
dorsal horn neurons at caudal region following spinal cord injury in rat. 2007.Online. Program No. 286.9. Ref Type: Abstract

23. Hains BC, Black JA, Waxman SG. Primary motor neurons fail to up-regulate voltage-gated sodium channel $\mathrm{Na}(\mathrm{v}) 1.3 /$ brain type III following axotomy resulting from spinal cord injury.

J.Neurosci.Res. 2002; 70:546-552. [PubMed: 12404508]

24. Hains BC, Waxman SG. Activated microglia contribute to the maintenance of chronic pain after spinal cord injury. J.Neurosci. 2006; 26:4308-4317. [PubMed: 16624951]

25. Hao JX, Xu XJ, Aldskogius H, Seiger A, Wiesenfeld-Hallin Z. Allodynia-like effects in rat after ischaemic spinal cord injury photochemically induced by laser irradiation. Pain. 1991; 45:175185. [PubMed: 1652116]

26. Harrison JK, Jiang Y, Chen S, Xia Y, Maciejewski D, McNamara RK, Streit WJ, Salafranca MN, Adhikari S, Thompson DA, Botti P, Bacon KB, Feng L. Role for neuronally derived fractalkine in mediating interactions between neurons and CX3CR1-expressing microglia.

Proc.Natl.Acad.Sci.U.S.A. 1998; 95:10896-10901. [PubMed: 9724801]

27. Hofstetter CP, Schweinhardt P, Klason T, Olson L, Spenger C. Numb rats walk - a behavioural and fMRI comparison of mild and moderate spinal cord injury. Eur.J.Neurosci. 2003; 18:3061-3068. [PubMed: 14656301]

28. Hubscher $\mathrm{CH}$. Ascending spinal pathways from sexual organs: effects of chronic spinal lesions. Prog.Brain Res. 2006; 152:401-414. [PubMed: 16198716]

29. Hutchinson KJ, Gomez-Pinilla F, Crowe MJ, Ying Z, Basso DM. Three exercise paradigms differentially improve sensory recovery after spinal cord contusion in rats. Brain. 2004; 127:14031414. [PubMed: 15069022]

30. Jakeman LB, Guan Z, Wei P, Ponnappan R, Dzwonczyk R, Popovich PG, Stokes BT. Traumatic spinal cord injury produced by controlled contusion in mouse. J.Neurotrauma. 2000; 17:299-319. [PubMed: 10776914]

31. Jin SX, Zhuang ZY, Woolf CJ, Ji RR. p38 mitogen-activated protein kinase is activated after a spinal nerve ligation in spinal cord microglia and dorsal root ganglion neurons and contributes to the generation of neuropathic pain. J.Neurosci. 2003; 23:4017-4022. [PubMed: 12764087]

32. Johnston IN, Milligan ED, Wieseler-Frank J, Frank MG, Zapata V, Campisi J, Langer S, Martin D, Green P, Fleshner M, Leinwand L, Maier SF, Watkins LR. A role for proinflammatory cytokines and fractalkine in analgesia, tolerance, and subsequent pain facilitation induced by chronic intrathecal morphine. J.Neurosci. 2004; 24:7353-7365. [PubMed: 15317861]

33. Kane CJ, Sims TJ, Gilmore SA. Astrocytes in the aged rat spinal cord fail to increase GFAP mRNA following sciatic nerve axotomy. Brain Res. 1997; 759:163-165. [PubMed: 9219877]

34. Kigerl KA, Lai W, Rivest S, Hart RP, Satoskar AR, Popovich PG. Toll-like receptor (TLR)-2 and TLR-4 regulate inflammation, gliosis, and myelin sparing after spinal cord injury. J.Neurochem. 2007; 102:37-50. [PubMed: 17403033]

35. Kim K, Yoon YW, Chung JM. Comparison of three rodent neuropathic pain models. Exp.Brain Res. 1997; 113:200-206. [PubMed: 9063706]

36. Kim SH, Chung JM. An experimental model for peripheral neuropathy produced by segmental spinal nerve ligation in the rat. Pain. 1992; 50:355-363. [PubMed: 1333581]

37. Kloos AD, Fisher LC, Detloff MR, Hassenzahl DL, Basso DM. Stepwise motor and all-or-none sensory recovery is associated with nonlinear sparing after incremental spinal cord injury in rats. Exp.Neurol. 2005; 191:251-265. [PubMed: 15649480]

38. Koshinaga M, Whittemore SR. The Temporal and Spatial Activation of Microglia in Fiber Tracts Undergoing Anterograde and Retrograde Degeneration Following Spinal Cord Lesion. J.Neurotrauma. 1995; 12:209-222. [PubMed: 7629867]

39. Laughlin TM, Bethea JR, Yezierski RP, Wilcox GL. Cytokine involvement in dynorphin-induced allodynia. Pain. 2000; 84:159-167. [PubMed: 10666520]

40. Ledeboer A, Sloane EM, Milligan ED, Frank MG, Mahony JH, Maier SF, Watkins LR. Minocycline attenuates mechanical allodynia and proinflammatory cytokine expression in rat models of pain facilitation. Pain. 2005; 115:71-83. [PubMed: 15836971] 
41. Lindia JA, McGowan E, Jochnowitz N, Abbadie C. Induction of CX3CL1 expression in astrocytes and CX3CR1 in microglia in the spinal cord of a rat model of neuropathic pain. J.Pain. 2005; 6:434-438. [PubMed: 15993821]

42. Lindsey AE, LoVerso RL, Tovar CA, Hill CE, Beattie MS, Bresnahan JC. An analysis of changes in sensory thresholds to mild tactile and cold stimuli after experimental spinal cord injury in the rat. Neurorehabil.Neural Repair. 2000; 14:287-300. [PubMed: 11402879]

43. Liu D, Thangnipon W, McAdoo DJ. Excitatory amino acids rise to toxic levels upon impact injury to the rat spinal cord. Brain Res. 1991; 547:344-348. [PubMed: 1884213]

44. Longbrake, E. Consequences of differential macrophage activation after spinal cord trauma. The Ohio State University; 2007. Ref Type: Thesis/Dissertation

45. Macias MY, Syring MB, Pizzi MA, Crowe MJ, Alexanian AR, Kurpad SN. Pain with no gain: allodynia following neural stem cell transplantation in spinal cord injury. Exp.Neurol. 2006; 201:335-348. [PubMed: 16839548]

46. McAdoo DJ, Xu GY, Robak G, Hughes MG. Changes in amino acid concentrations over time and space around an impact injury and their diffusion through the rat spinal cord. Exp.Neurol. 1999; 159:538-544. [PubMed: 10506525]

47. Milligan ED, Zapata V, Chacur M, Schoeniger D, Biedenkapp J, O'Connor KA, Verge GM, Chapman G, Green P, Foster AC, Naeve GS, Maier SF, Watkins LR. Evidence that exogenous and endogenous fractalkine can induce spinal nociceptive facilitation in rats. Eur.J.Neurosci. 2004; 20:2294-2302. [PubMed: 15525271]

48. Mills CD, Johnson KM, Hulsebosch CE. Group I metabotropic glutamate receptors in spinal cord injury: roles in neuroprotection and the development of chronic central pain. J.Neurotrauma. 2002; 19:23-42. [PubMed: 11852976]

49. Molander C, Xu Q, Grant G. Cytoarchitectonic organization of the spinal cord in the rat: I. The lower thoracic and lumbosacral cord. J.Comp.Neurol. 1984; 230:133-141. [PubMed: 6512014]

50. Nesic O, Lee J, Johnson KM, Ye Z, Xu GY, Unabia GC, Wood TG, McAdoo DJ, Westlund KN, Hulsebosch CE, Regino Perez-Polo J. Transcriptional profiling of spinal cord injury-induced central neuropathic pain. J.Neurochem. 2005; 95:998-1014. [PubMed: 16219025]

51. Peng XM, Zhou ZG, Glorioso JC, Fink DJ, Mata M. Tumor necrosis factor-alpha contributes to below-level neuropathic pain after spinal cord injury. Ann.Neurol. 2006; 59:843-851. [PubMed: 16634039]

52. Popovich PG, Wei P, Stokes BT. Cellular inflammatory response after spinal cord injury in Sprague-Dawley and Lewis rats. J.Comp.Neurol. 1997; 377:443-464. [PubMed: 8989657]

53. Raghavendra V, Tanga F, DeLeo JA. Inhibition of microglial activation attenuates the development but not existing hypersensitivity in a rat model of neuropathy. J.Pharmacol.Exp.Ther. 2003; 306:624-630. [PubMed: 12734393]

54. Raghavendra V, Tanga FY, DeLeo JA. Complete Freunds adjuvant-induced peripheral inflammation evokes glial activation and proinflammatory cytokine expression in the CNS. Eur.J.Neurosci. 2004; 20:467-473. [PubMed: 15233755]

55. Reier PJ, Houle JD. The glial scar: its bearing on axonal elongation and transplantation approaches to CNS repair. Adv.Neurol. 1988; 47:87-138. [PubMed: 3278533]

56. Siddall P, Xu CL, Cousins M. Allodynia following traumatic spinal cord injury in the rat. NeuroReport. 1995; 6:1241-1244. [PubMed: 7669978]

57. Siddall PJ, McClelland JM, Rutkowski SB, Cousins MJ. A longitudinal study of the prevalence and characteristics of pain in the first 5 years following spinal cord injury. Pain. 2003; 103:249-257. [PubMed: 12791431]

58. Sroga JM, Jones TB, Kigerl KA, McGaughy VM, Popovich PG. Rats and mice exhibit distinct inflammatory reactions after spinal cord injury. J.Comp.Neurol. 2003; 462:223-240. [PubMed: 12794745]

59. Stuesse SL, Crisp T, McBurney DL, Schechter JB, Lovell JA, Cruce WL. Neuropathic pain in aged rats: behavioral responses and astrocytic activation. Exp.Brain Res. 2001; 137:219-227. [PubMed: 11315551]

Exp Neurol. Author manuscript; available in PMC 2009 August 01. 
60. Sweitzer S, Martin D, DeLeo JA. Intrathecal interleukin-1 receptor antagonist in combination with soluble tumor necrosis factor receptor exhibits an anti-allodynic action in a rat model of neuropathic pain. Neuroscience. 2001; 103:529-539. [PubMed: 11246166]

61. Takeda K, Sawamura S, Sekiyama H, Tamai H, Hanaoka K. Effect of methylprednisolone on neuropathic pain and spinal glial activation in rats. Anesthesiology. 2004; 100:1249-1257. [PubMed: 15114224]

62. Tanga FY, Nutile-McMenemy N, DeLeo JA. The CNS role of Toll-like receptor 4 in innate neuroimmunity and painful neuropathy. PNAS. 2005; 102:5856-5861. [PubMed: 15809417]

63. Tanga FY, Raghavendra V, DeLeo JA. Quantitative real-time RT-PCR assessment of spinal microglial and astrocytic activation markers in a rat model of neuropathic pain. Neurochem.Int. 2004; 45:397-407. [PubMed: 15145554]

64. Tanga FY, Raghavendra V, Nutile-McMenemy N, Marks A, DeLeo JA. Role of astrocytic S100beta in behavioral hypersensitivity in rodent models of neuropathic pain. Neuroscience. 2006; 140:1003-1010. [PubMed: 16600520]

65. Tawfik VL, Nutile-McMenemy N, Lacroix-Fralish ML, DeLeo JA. Efficacy of propentofylline, a glial modulating agent, on existing mechanical allodynia following peripheral nerve injury. Brain Behav Immun. 2007; 21:238-246. [PubMed: 16949251]

66. Tsuda M, Shigemoto-Mogami Y, Koizumi S, Mizokoshi A, Kohsaka S, Salter MW, Inoue K. P2X4 receptors induced in spinal microglia gate tactile allodynia after nerve injury. Nature. 2003; 424:778-783. [PubMed: 12917686]

67. Viviani B, Bartesaghi S, Corsini E, Galli CL, Marinovich M. Cytokines role in neurodegenerative events. Toxicology Letters. 2004; 149:85-89. [PubMed: 15093252]

68. Widerstrom-Noga EG, Felipe-Cuervo E, Yezierski RP. Chronic pain after spinal injury: interference with sleep and daily activities. Arch.Phys.Med.Rehabil. 2001; 82:1571-1577. [PubMed: 11689978]

69. Yezierski RP. Pain following spinal cord injury: Pathophysiology and central mechanisms. Prog.Brain Res. 2000; 129:429-449. [PubMed: 11098709]

70. Yezierski RP, Park SH. The mechanosensitivity of spinal sensory neurons following intraspinal injections of quisqualic acid in the rat. Neurosci.Lett. 1993; 157:115-119. [PubMed: 8233021]

71. Zhang YH, Lin JX, Vilcek J. Interleukin-6 induction by tumor necrosis factor and interleukin-1 in human fibroblasts involves activation of a nuclear factor binding to a kappa Blike sequence. Molecular and Cellular Biology. 1990; 10:3818-3823. [PubMed: 2192263]

72. Zhao ML, Kim MO, Morgello S, Lee SC. Expression of inducible nitric oxide, interleukin-1 and caspase-1 in HIV-1 encephalitis. Journal of Neuroimmmunology. 2001; 115:182-191.

73. Zhao P, Waxman SG, Hains BC. Extracellular signal-regulated kinase-regulated microglia-neuron signaling by prostaglandin E2 contributes to pain after spinal cord injury. J.Neurosci. 2007a; 27:2357-2368. [PubMed: 17329433]

74. Zhao P, Waxman SG, Hains BC. Modulation of thalamic nociceptive processing after spinal cord injury through remote activation of thalamic microglia by cysteine cysteine chemokine ligand 21 . J.Neurosci. 2007b; 27:8893-8902. [PubMed: 17699671]

75. Zhuang ZY, Wen YR, Zhang DR, Borsello T, Bonny C, Strichartz GR, Decosterd I, Ji RR. A Peptide c-Jun N-Terminal Kinase (JNK) Inhibitor Blocks Mechanical Allodynia after Spinal Nerve Ligation: Respective Roles of JNK Activation in Primary Sensory Neurons and Spinal Astrocytes for Neuropathic Pain Development and Maintenance. J.Neurosci. 2006; 26:3551-3560. [PubMed: 16571763]

Exp Neurol. Author manuscript; available in PMC 2009 August 01. 


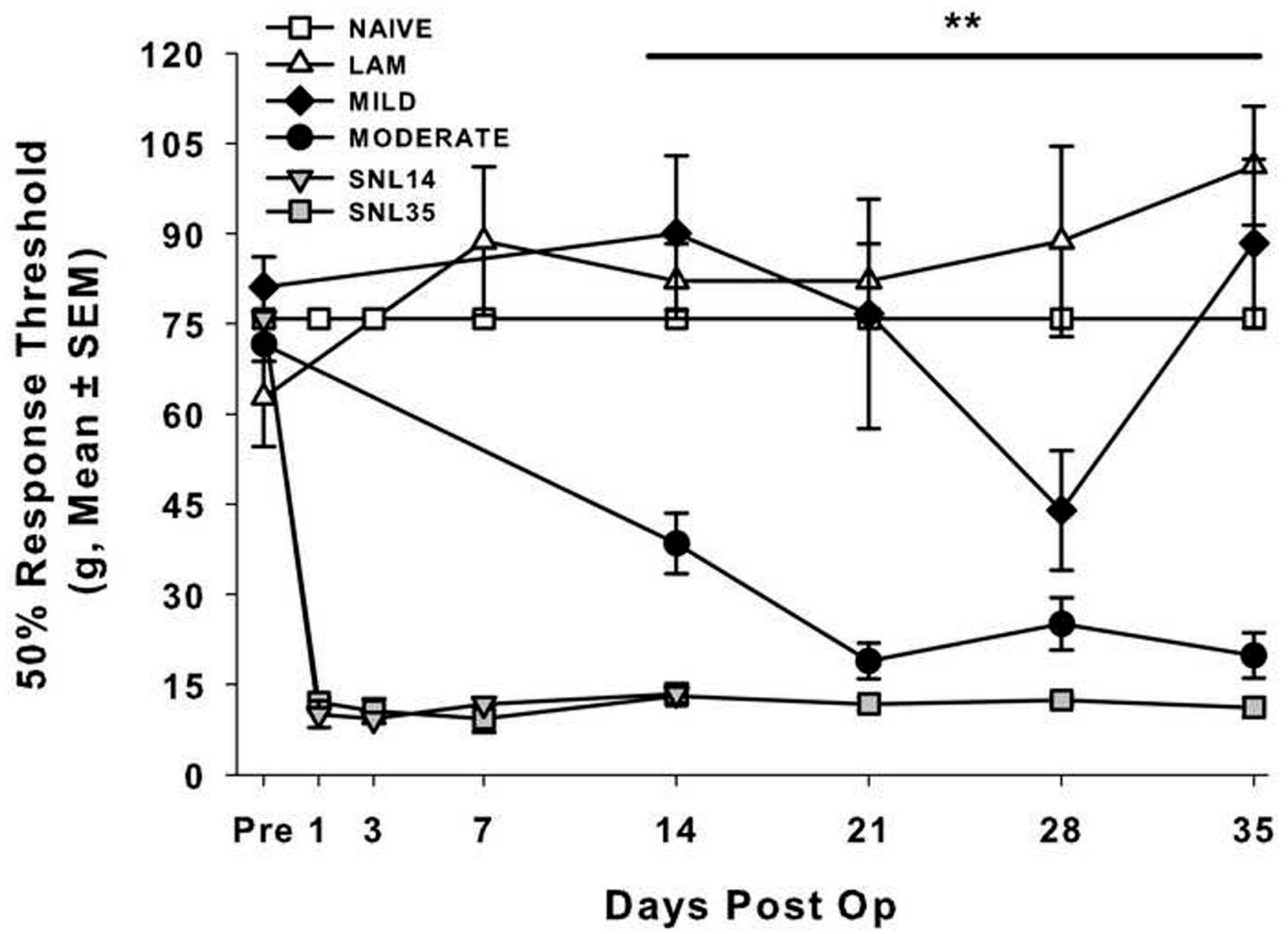

Figure 1.

Pain-like sensory thresholds occurred after moderate SCI and modified SNL. Using 50\% withdrawal to VFH stimulation, significantly lower sensory thresholds of the hindpaw occurred for moderate SCI and mSNL groups from 14 to 35 dpo. Naïve, laminectomy and mild SCI groups had normal sensory thresholds throughout the testing period. (Significance determined by repeated measures ANOVA; **p<.05 except at $28 \mathrm{dpo}$ ). Note that an equivalent severity of allodynia occurred between moderate SCI and $\mathrm{mSNL}$, a wellestablished peripheral model of neuropathic pain. 

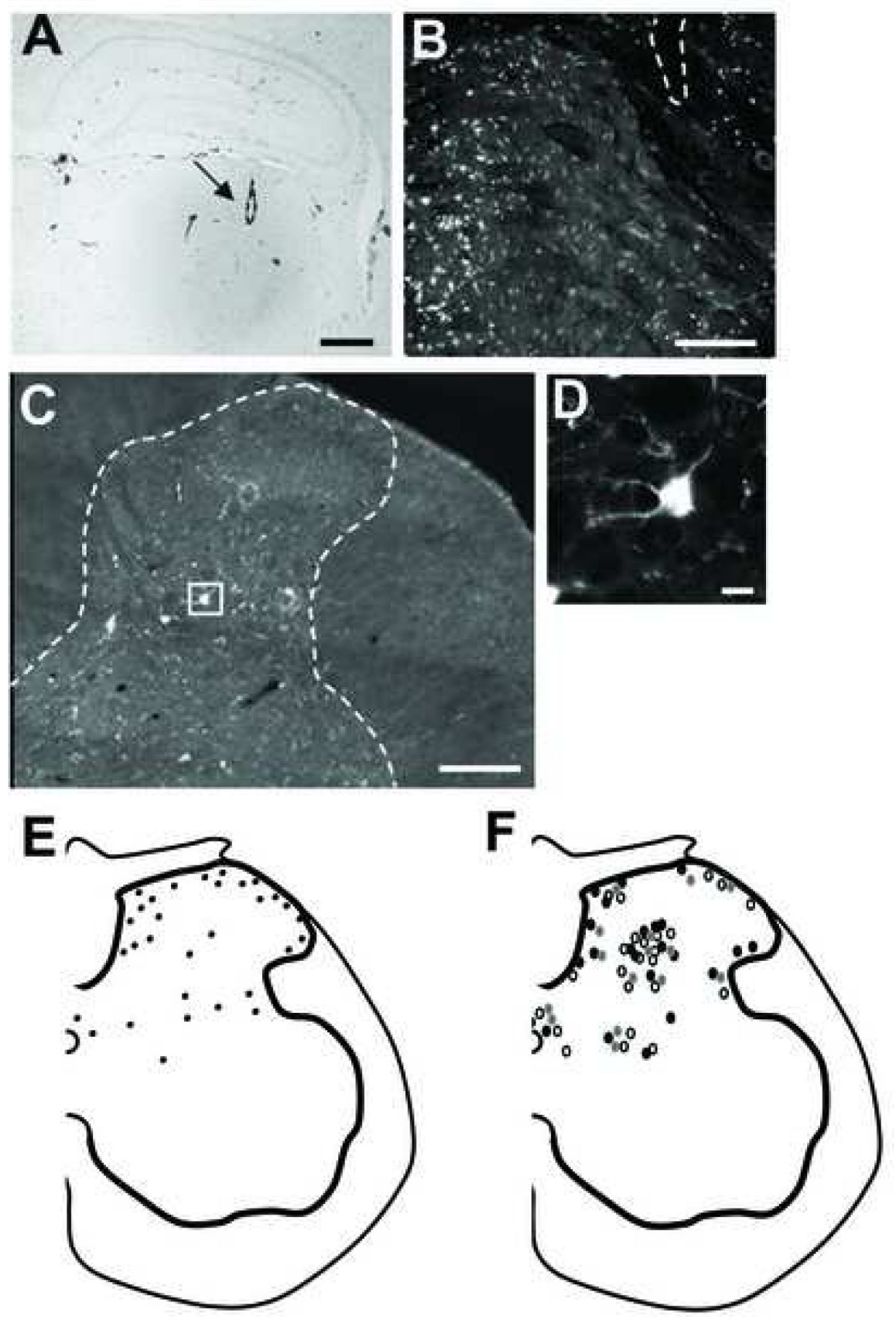

Figure 2.

Neuroanatomical substrates of the spinothalamic tract after moderate SCI. The ventroposterolateral (VPL) nucleus of the thalamus was injected with $2 \%$ fluorogold (FG) in rats with allodynia induced by moderate $\mathrm{SCI}(\mathrm{n}=3)$. The $\mathrm{FG}$ injection was localized to the hindlimb region of the VPL visualized with brightfield (A) and fluorescence (B; dashed line indicates the injection track). FG-positive neurons were distributed in the L5 dorsal horn (C). Positive neuronal labeling included bright opacity of the cytoplasm and proximal dendrites (D; high power image of cell outlined by the square in C). The L5 spinothalamic neurons 
projecting to the VPL in a Naïve rat are distributed in the superficial and deep dorsal horn (E). The profiles reflect FG+ neurons in two sections $700 \mu \mathrm{m}$ apart in the L5 dorsal horn. A similar distribution of spinothalamic neurons occurred after moderate SCI (F). Three SCI rats with allodynia are represented by different symbols for neuronal profiles in two sections $700 \mu \mathrm{m}$ apart $(\mathrm{F})$. Note that the majority of spared spinothalamic axons in allodynic rats project from the deep rather than superficial dorsal horn. [ Scale bars: $A=1 \mathrm{~mm} ; \mathrm{B}, \mathrm{C}=300$ $\mu \mathrm{m} ; \mathrm{D}=50 \mu \mathrm{m}]$ 
A

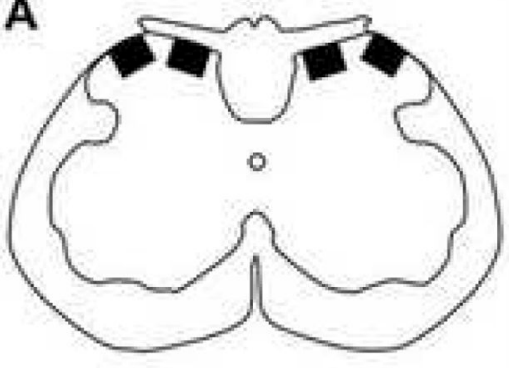

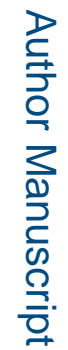
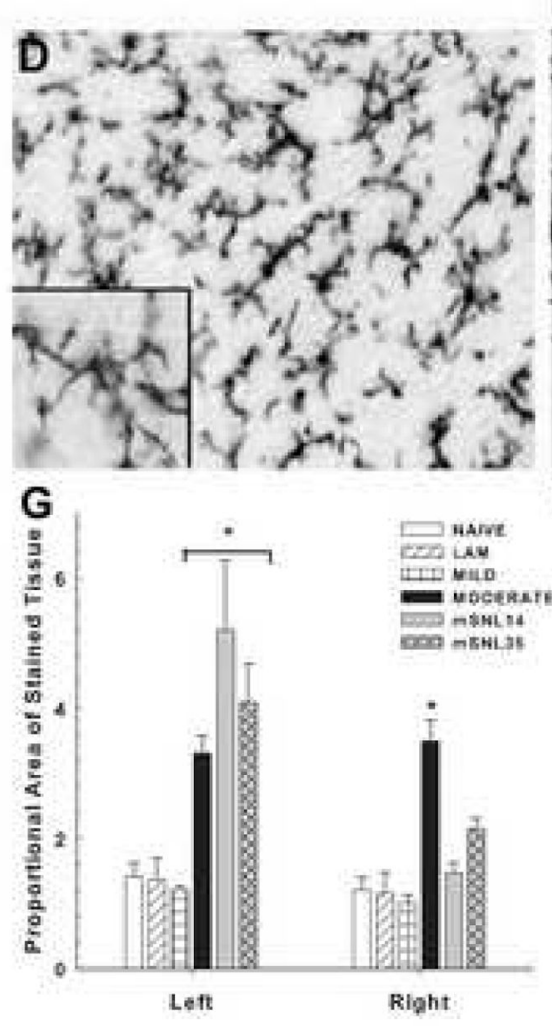
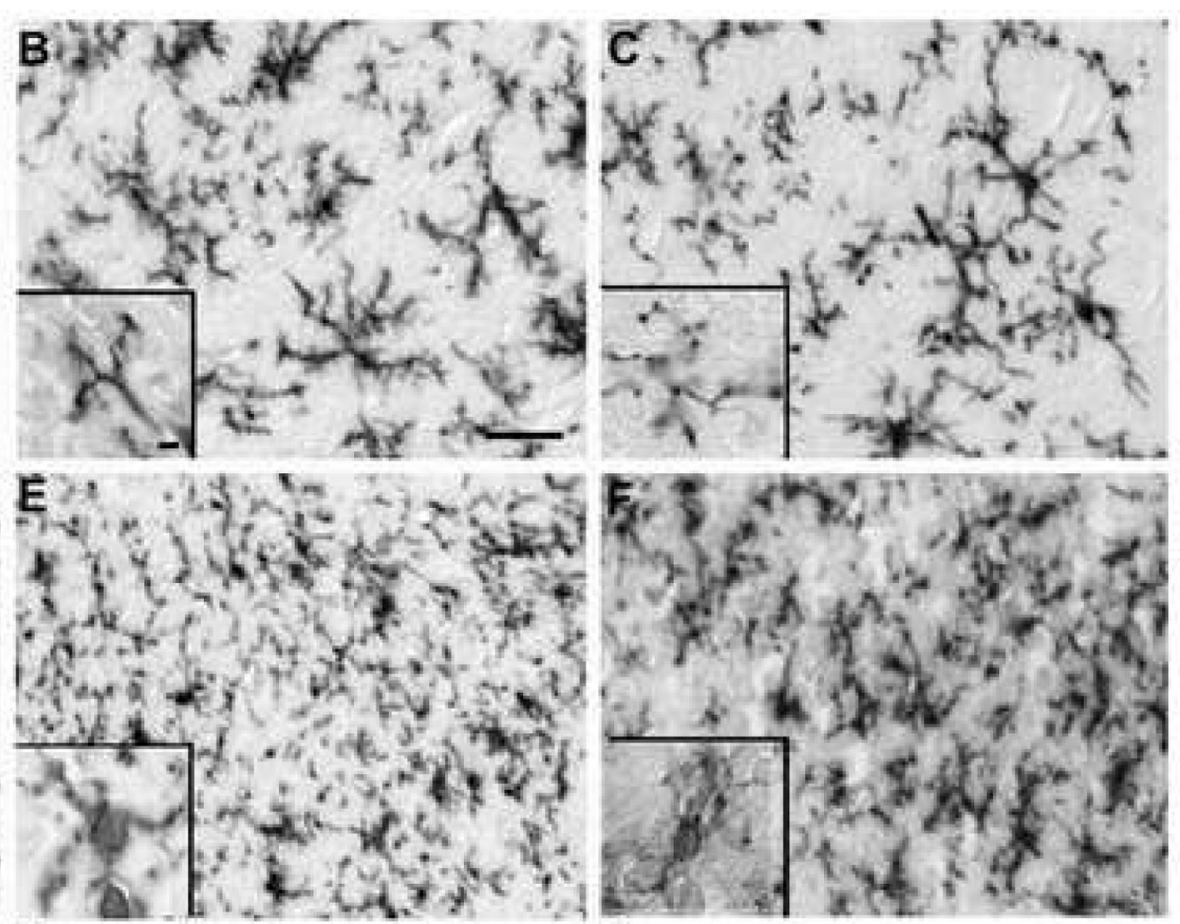

$\mathrm{H}$

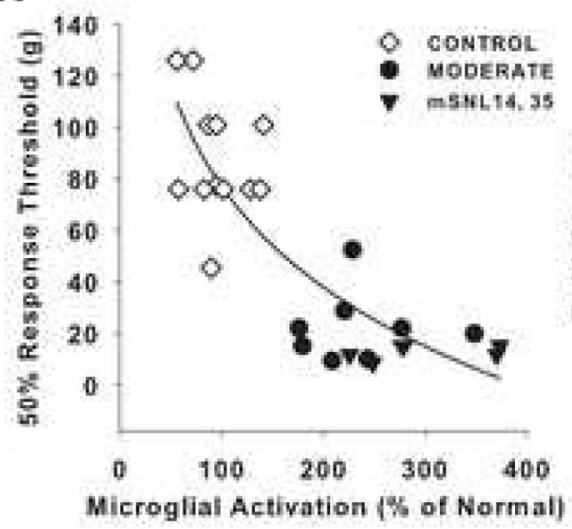

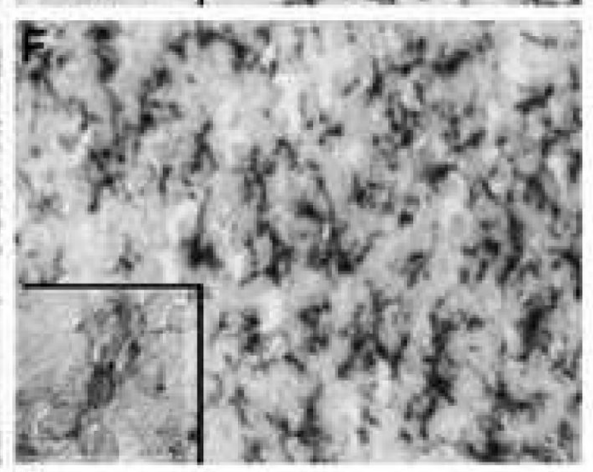

I

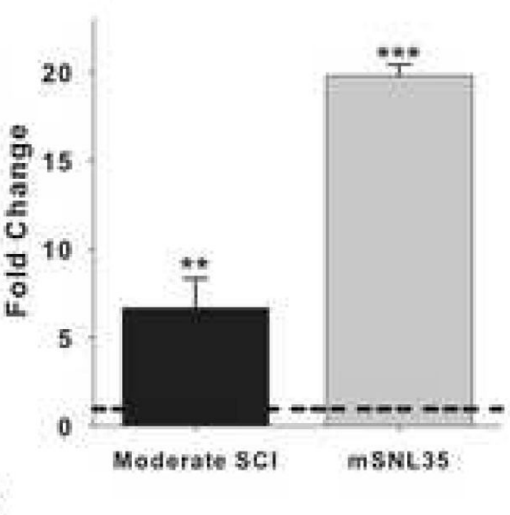

Figure 3.

Immunolabeling and quantification of activated and resting microglia within the superficial dorsal horn. Basal levels of OX42 signal were observed within the L5 dorsal horn of Naïve (B) and Mild SCI (C) rats. Higher magnification displays the characteristic resting phenotype of microglia with slender processes and small cell body for naïve (inset of B) and mild SCI (inset C). After Moderate SCI (D) and spinal nerve ligation (mSNL14, E; mSNL35, F), microglia exhibited an activated phenotype with retracted, thickened processes and large cell bodies (high magnification insets D, E, F). Proportional area was measured within 2 regions of interest $\left(0.026 \mathrm{~mm}^{2}\right)$ aligned with anatomical landmarks along the 
medial and lateral portion of the dorsal horn as shown in A. The summed proportional areas for these regions of interest are represented in G. Microglial activation was significantly greater ipsilateral to $\mathrm{mSNL}$ (left) and bilaterally after Moderate SCI (right and left) compared to Mild SCI, naïve and laminectomy (LAM) controls (G). The degree of microglial activation predicted allodynia (H). Microglial activation of $200 \%$ or higher was associated with the development of mechanical allodynia $\left(\mathrm{H} ; \mathrm{r}^{2}=0.83 ; \mathrm{p}<.01\right)$. Rats without microglial activation in the L5 dorsal horn displayed normal sensory thresholds (controls; Naïve, Laminectomy or Mild SCI). Using LCM/rtPCR detection of microglial marker CD11b (I) distinguished increased microglial message at 35 days after moderate SCI or mSNL compared to naïve (dashed line). [Significance in G: repeated measures ANOVA $* \mathrm{p}<.05$ vs. Naïve, LAM and Mild SCI. Significance in I: one way ANOVA ** p<.01; *** $\mathrm{p}<.001]$. All histological sections are of the medial box in the left dorsal horn. Scale Bar B$\mathrm{F}=200 \mu \mathrm{m}$; Inset Scale bar $=10 \mu \mathrm{m}$. 

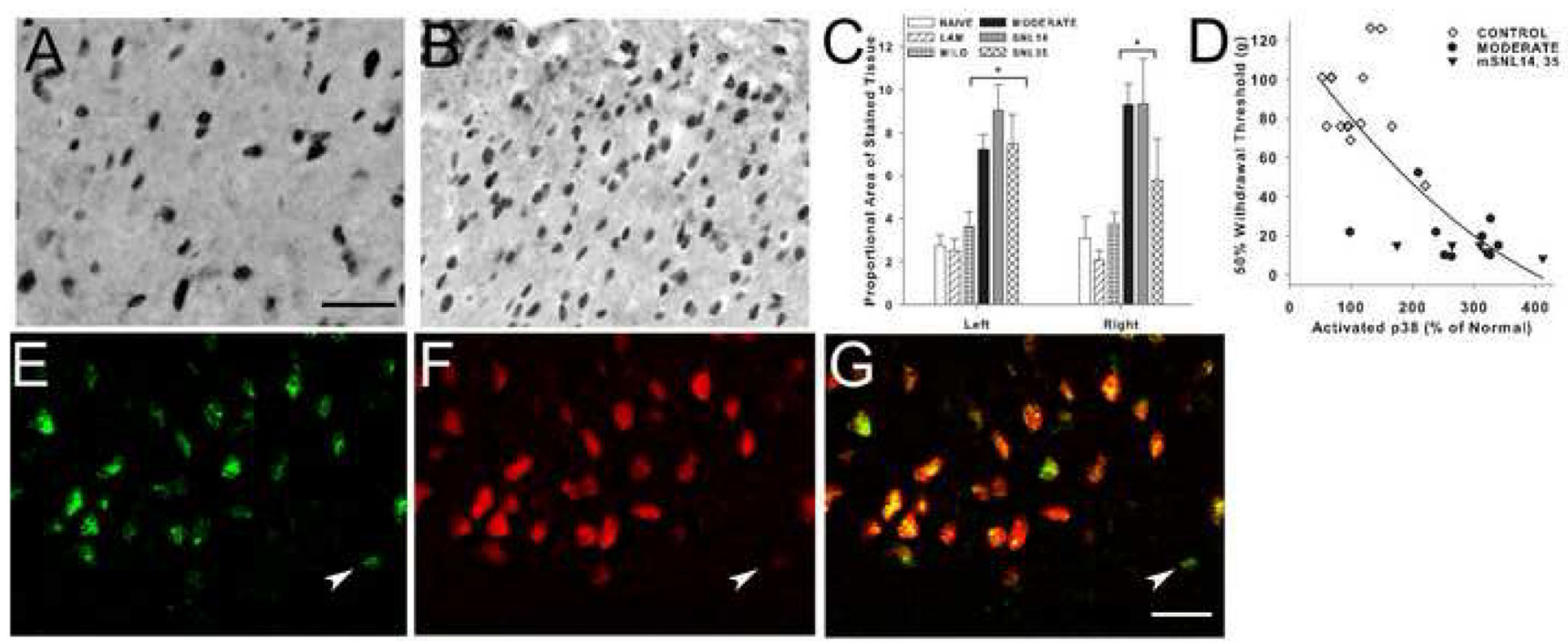

Figure 4.

Immunolabeling and quantification of phosphorylated p38 in the L5 superficial dorsal horns. Representative section of the left L5 dorsal horns of Naïve (A) and Moderate SCI (B) at 35 dpo. Proportional area measurements within regions of interest depicted in figure $3 \mathrm{~A}$ showed that rats with Moderate SCI and mSNL at short (14 day,) and long (35 day) survival had greater activated $\mathrm{p} 38$ protein compared to Naïve, Laminectomy (LAM) or Mild SCI groups (C). Phosphorylated p38 MAP kinase correlated to neuropathic pain (D, Pearson Coefficient $\left.=-0.78 ; \mathrm{p}<.01 ; \mathrm{r}^{2}=0.634\right)$. Double-label immunofluorescence with p-p38 (E) and NeuN $(F)$ antibodies, showed that $\mathrm{p} 38$ MAPK activation occurs predominantly in neurons $(\mathrm{G}$; arrowhead denotes a p-p38+, NeuN- cell). (Significance in C: repeated measures ANOVA* $\mathrm{p}<.05$ vs Naive, LAM and Mild SCI). Scale Bar A and B $=200 \mu \mathrm{m}$. Scale Bar E, F, G = $20 \mu \mathrm{m}$. 

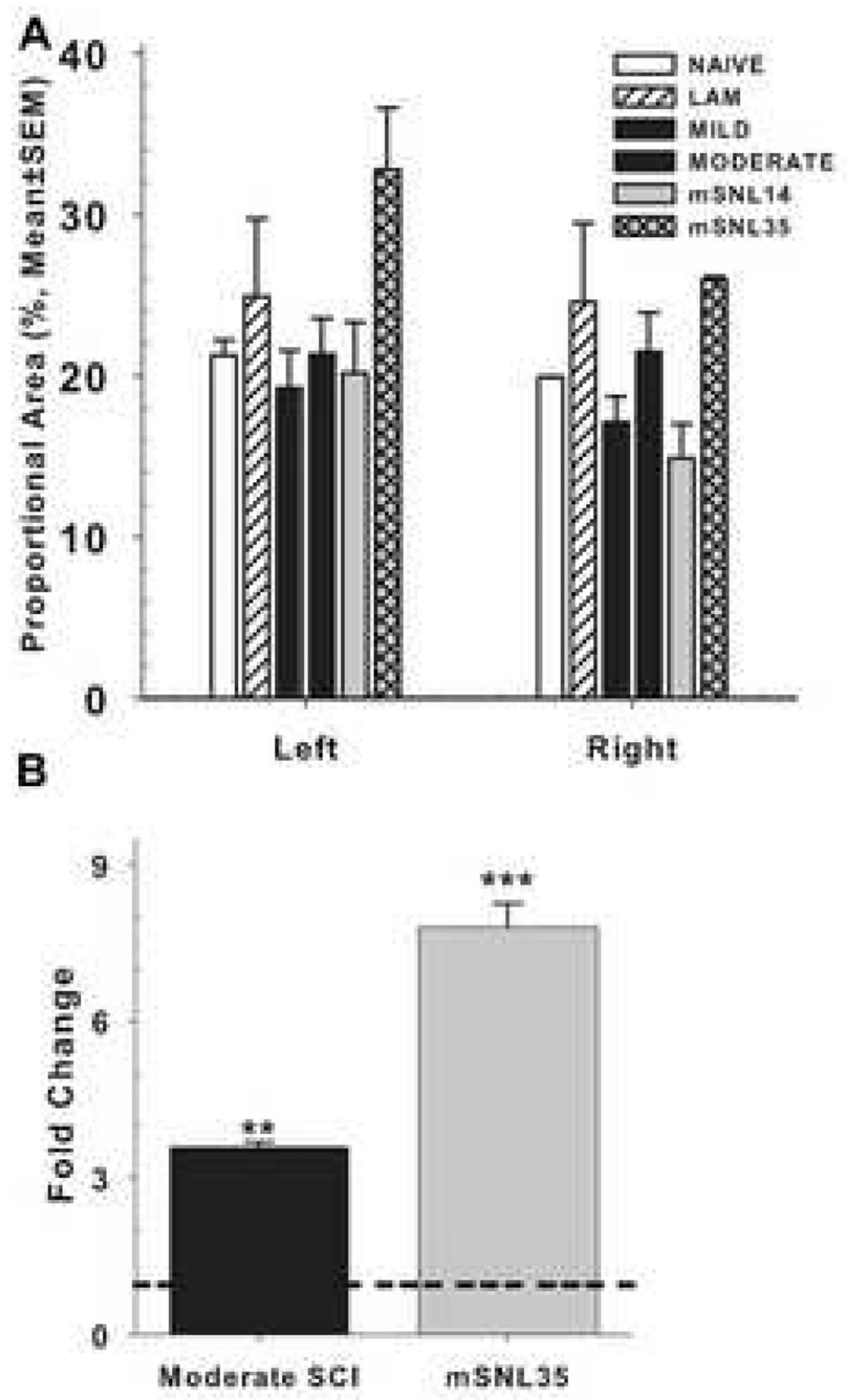

Figure 5.

Quantification of reactive astrocytes at L5. Basal levels of GFAP signal were observed within the dorsal and ventral horn of L5 spinal cord regardless of injury. Proportional area of positively labeled tissue throughout the medial-lateral span of the superficial dorsal horn (A) showed no increase above naïve and laminectomy (LAM) controls after mild or moderate SCI or modified spinal nerve ligation despite a significant increase in GFAP mRNA in SCI and mSNL groups relative to naive(dashed line; B). (Significance in B: one way ANOVA $* * \mathrm{p}<.01 ; * * * \mathrm{p}<.001)$. 

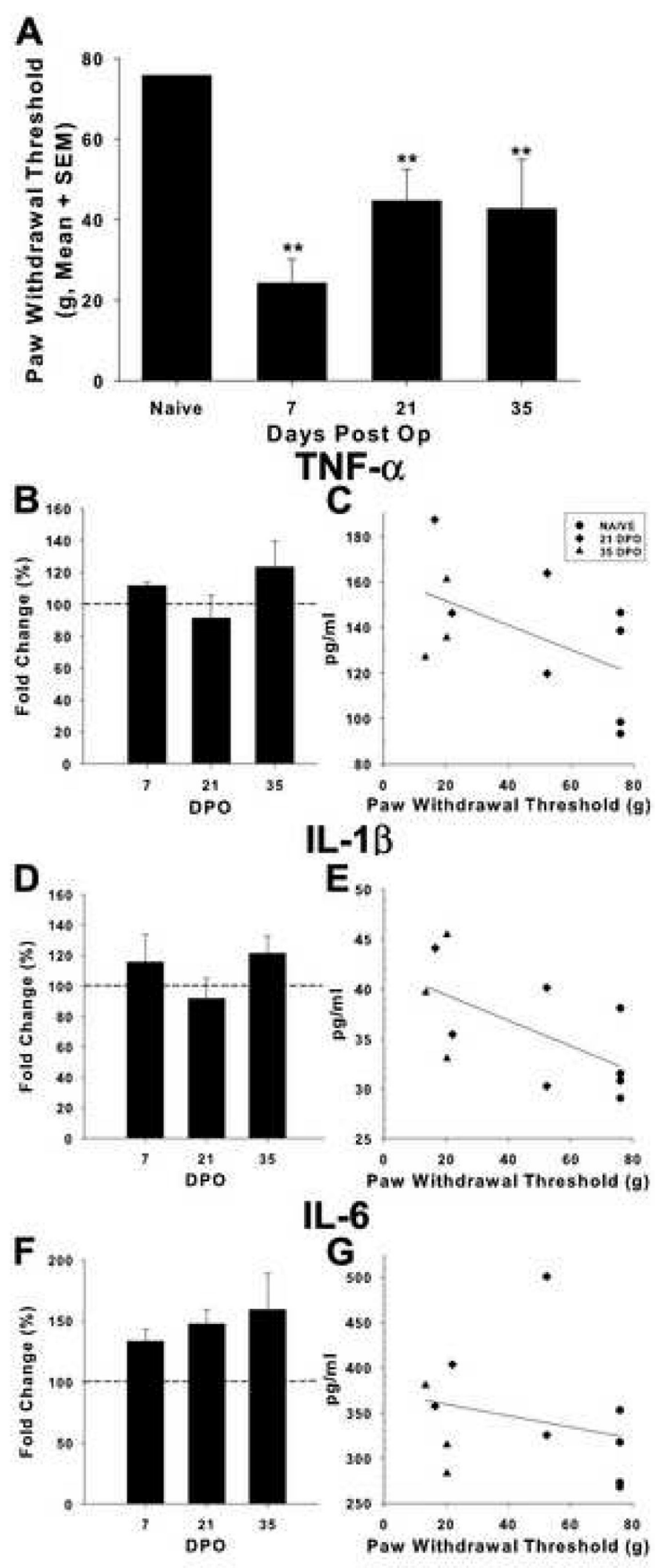

Figure 6.

Analysis of pro-inflammatory cytokine levels in the L5 dorsal spinal cord of rats with allodynia. Stimulation with VFH to the dorsal region of the L5 dermatome detected significantly lower sensory thresholds from 7 to 35 days after moderate SCI (A). Rats with pain-like behavior demonstrated significantly greater tumor necrosis factor-a (TNF-a, B) and interleukin (IL)-1 $\beta$ (D) protein early after injury, with levels returning to normal by 35 dpo. IL-6 protein (F) increased at later time points after SCI when pain-like behavior was fully established. Thus, TNF- $a$ and IL- $1 \beta$ may play a role in the induction of pain-like 
behavior while IL-6 may be involved in pain maintenance. These increases in proinflammatory cytokines correlate to pain-like behavior as determined by a Spearmann Rank Test ( $\mathrm{p}<.05 ; \mathrm{C}, \mathrm{E}, \mathrm{G})$. Greater cytokine proteins in the dorsal spinal cord predicted lower L5 sensory thresholds. Naïve expression levels are denoted as dashed lines (B, D, F). (Significance: one way ANOVA *p<.05; **p<.01 vs. Naïve). 

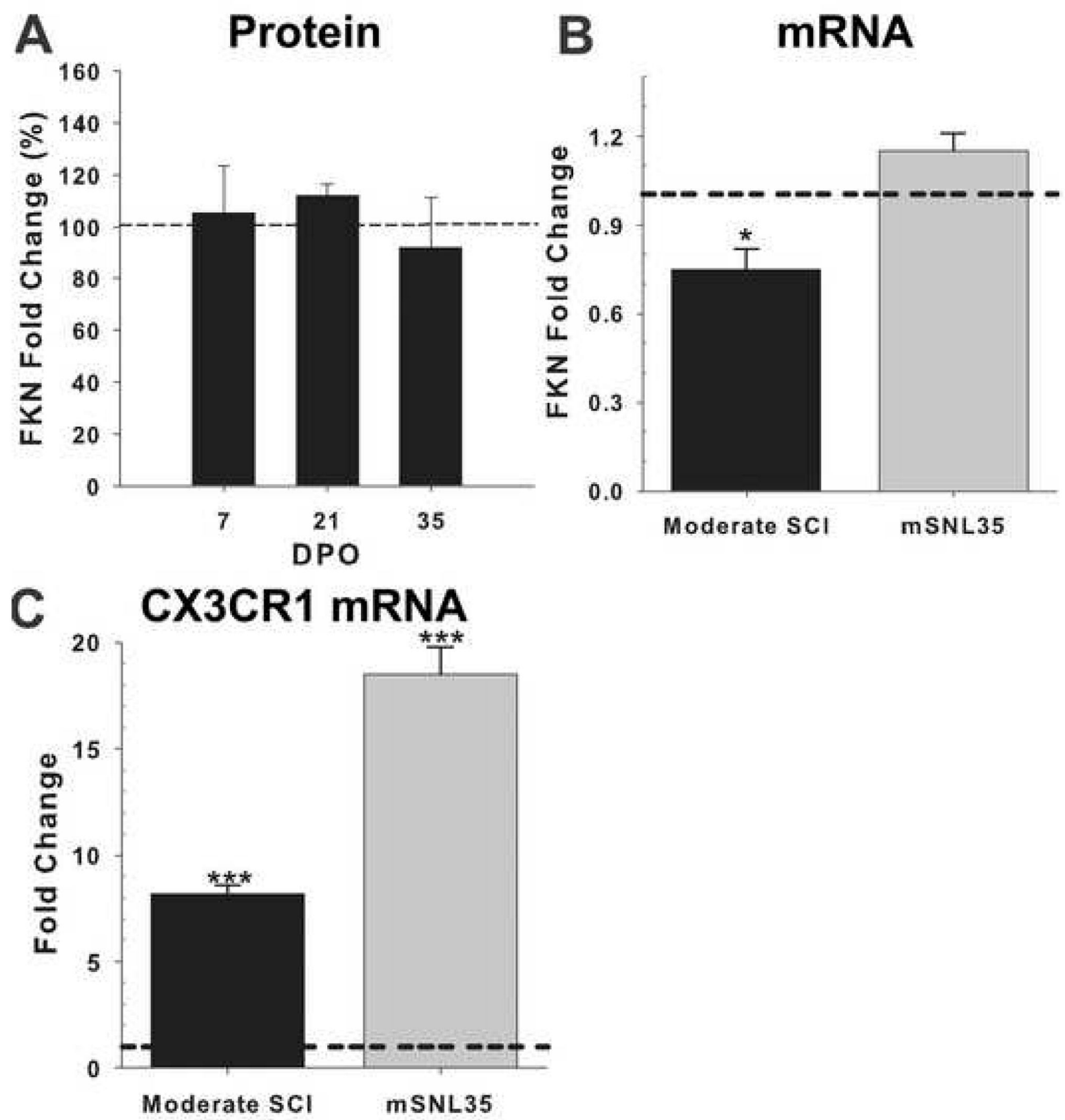

Figure 7.

Fractalkine (FKN) protein levels were unchanged in the L5 dorsal spinal cord of rats with SCI-induced allodynia over time (A). Interestingly, fractalkine mRNA did not increase above naïve levels after spinal nerve ligation and was significantly lower for moderate SCI at 35 dpo (B). The fractalkine receptor, CX3CR1, showed at least an eight-fold increase in message after moderate SCI and more than a 15 -fold increase after mSNL at $35 \mathrm{dpo}$ (C). 
Naïve expression levels are denoted as dashed lines. (Significance: one way ANOVA $* \mathrm{p}<.05 ; * * \mathrm{p}<.01 ; * * * \mathrm{p}<.001$ vs. naïve). 
Table 1

List of forward and reverse primer sequences used for RT-PCR.

\begin{tabular}{|l|l|l|l|}
\hline Primer & Forward & Reverse & Concentration \\
\hline CD11b & ATGCTTCTGCAGTCATCTCGAG & TATCGAGGTGCCCCTAAAACC & $100 \mathrm{nM}$ \\
\hline CX3CR1 & GTGACATCGTGGCCTTTGG & CCAGACCGAACGTGAAGACA & $500 \mathrm{nM}$ \\
\hline FKN & TCCTACATCTGAGAGCGCAGTTC & AGTTCTGGCCCTGCCTGAA & $500 \mathrm{nM}$ \\
\hline GAPDH & CGGATTTGGCCGTATCG & CAATGTCCACTTTGTCACAAGAGA & $500 \mathrm{nM}$ \\
\hline GFAP & AGCCTGGACACCAAATCTGTG & CCGTCTTTACCACGATGTTCC & $100 \mathrm{nM}$ \\
\hline P2RX4 & TGACAACCAAAGCCAAAGGTG & ATCCGGAATCCAAGCTGAGAG & $500 \mathrm{nM}$ \\
\hline P38a & AGCAGATAATGCGTCTGACGG & GGCTTGGCATCCTGTTAATGAG & $500 \mathrm{nM}$ \\
\hline
\end{tabular}

